\title{
CONOCIMIENTOS Y PRÁCTICAS SOBRE LAS MEDIDAS DE CONTROL DE INFECCIÓN POR TUBERCULOSIS EN UNA INSTITUCIÓN DE SALUD
}

Recibido: noviembre del 2018

Aceptado: abril del 2019

\author{
Alba Idaly Muñoz Sánchez ${ }^{1}$, Carlos Enrique Awad García², \\ Carlos Julio Saavedra Cantor ${ }^{3}$
}

\section{Resumen}

Introducción: Algunos trabajadores de la salud tienen conocimientos y prácticas erróneas sobre las medidas de control de tuberculosis, lo que aumenta su probabilidad de contagio en las instituciones de salud. Objetivo: Describir los conocimientos y las prácticas sobre las medidas de control de infección por tuberculosis en trabajadores de la salud antes y después de una intervención educativa. Métodos: Estudio de tipo evaluativo con modelo antesdespués. El proyecto tuvo una duración de 12 meses. Se aplicó un pre-test a los trabajadores de la salud, se diseñó una intervención educativa sustentada en la andragogía y se aplicó un pos-test seis meses después. Se utilizaron estadísticos descriptivos, se realizó la prueba no paramétrica de McNemar para muestras relacionadas o dependientes. Resultados: Participaron 216 trabajadores de la salud. Se encontró un aumento significativo después de la intervención educativa sobre los conocimientos y las prácticas de las medidas de control de tuberculosis en aspectos como la prueba de tuberculina $(\mathrm{P}<0,05)$, la captación de sintomáticos respiratorios $(\mathrm{P}<0,05)$, ventilación natural cruzada $(\mathrm{P}<0,05)$, ventilación mecánica $(\mathrm{P}<0,05)$, mascarilla de alta eficiencia $\mathrm{N} 95(\mathrm{P}<0,05)$ y la implementación de los pasos adecuados para usar este elemento de protección personal $(\mathrm{P}<0,05)$. Conclusión: La intervención educativa contribuyó a mejorar los conocimientos y las prácticas de los trabajadores de la salud sobre las medidas de control de la infección por tuberculosis. Las intervenciones educativas pueden contribuir al control de la tuberculosis en las instituciones de salud.

Palabras clave: control de infecciones, instituciones de salud, personal de salud, tuberculosis.

${ }^{1}$ Enfermera, especialista en Salud Ocupacional, magíster y doctora en Enfermería. Profesora titular de la Universidad Nacional de Colombia, Grupo de Investigación Salud y Cuidado de los Colectivos.Correo: aimunozs@unal.edu.co

2 Médico, especialista en Medicina Interna y Neumología. Subred Integrada de Servicios de Salud Centro Oriente, Unidad Especializada de Servicios de Salud Santa Clara. Correo: carlosawad@gmail.com

${ }^{3}$ Enfermero, especialista en Salud Ocupacional. Universidad Nacional de Colombia, Grupo de Investigación Salud y Cuidado de los Colectivos. Correo: cajsaavedraca@unal.edu.co 


\section{KNOWLEDGE AND PRACTICES REGARDING CONTROL MEASUREMENTS OF INFECTION WITH TUBERCULOSIS IN A HEALTH INSTITUTION}

Alba Idaly Muñoz Sánchez¹ , Carlos Enrique Awad García²,

Carlos Julio Saavedra Cantor ${ }^{3}$

\section{Abstract}

Introduction: Some health workers have knowledge and wrong practices regarding the measurements of control of tuberculosis, which increases the probability of infection in health institutions. Objective: To describe knowledge and practices regarding the control measurements for infection with tuberculosis in workers in the health areas, before and after an educational intervention. Method: Assessment study with a pre and post model. The project lasted 12 months. We applied a previous test to the workers, then we designed an intervention based in andragogy and we applied an after-test, six months later. We used descriptive statistics and we carried out the non-parametric McNemar test for samples related or dependent. Results: 216 health workers took part. We found out a meaningful increase after the pedagogical intervention regarding the knowledge and practice of the control measurements of tuberculosis in aspects as the tuberculine test $(\mathrm{P}<0,05)$, the testing of respiratory symptoms ( $\mathrm{P}$ $<0,05)$, crossed natural ventilation $(P<0,05)$, mechanic ventilation ( $P$ $<0,05)$, high efficiency mask N95 $(\mathrm{P}<0,05)$ and the implementation of adequate steps to use this element of personal protection. $(\mathrm{P}<$ 0,05). Conclusión: The pedagogical intervention contributed to improve knowledge and practices of health workers regarding the control measurements for the infection with tuberculosis. Educational interventions may contribute to the control of tuberculosis in health institutions. 


\section{CONHECIMENTOS E PRÁTICAS SOBRE AS MEDIDAS DE CONTROLE DE INFECÇÃO POR TUBERCULOSE EM UMA INSTITUIÇÃO DE SAÚDE}

Alba Idaly Muñoz Sánchez ${ }^{1}$, Carlos Enrique Awad García²,

Carlos Julio Saavedra Cantor ${ }^{3}$

\section{Resumo}

Introdução: alguns profissionais da saúde têm conhecimentos e práticas equivocadas sobre as medidas de controle da tuberculose, o que aumenta sua probabilidade de contágio nas instituições de saúde. Objetivo: descrever os conhecimentos e as práticas sobre as medidas de controle de infecção por tuberculose em trabalhadores da saúde antes e depois de uma intervenção educativa. Métodos: estudo de tipo avaliativo com modelo antes-depois. $\mathrm{O}$ projeto durou 12 meses. Foi aplicado um pré-teste aos trabalhadores da saúde; foi desenhada uma intervenção educativa sustentada na andragogia e foi aplicado um pós-teste seis meses depois. Foram utilizados indicadores estatísticos descritivos e realizado um teste não paramétrico de McNemar para amostras relacionadas ou dependentes. Resultados: participaram 216 profissionais da saúde. Foi verificado aumento significativo depois da intervenção educativa sobre os conhecimentos e as práticas das medidas de controle de tuberculose em aspectos como o exame de tuberculina $(\mathrm{P}<0,05)$, a captação de sintomáticos respiratórios $(\mathrm{P}<0,05)$, a ventilação natural cruzada $(\mathrm{P}<0,05)$, a ventilação mecânica $(\mathrm{P}<0,05)$, a máscara de alta eficiência N95 $(\mathrm{P}<0,05)$ e a implantação dos passos adequados para usar esse elemento de proteção pessoal $(\mathrm{P}<$ 0,05). Conclusão: a intervenção educativa contribuiu para melhorar os conhecimentos e as práticas dos profissionais da saúde sobre as medidas de controle da infecção por tuberculose. As intervenções educativas podem ajudar no controle da tuberculose nas instituições de saúde. 


\section{Introducción}

La tuberculosis es una enfermedad infectocontagiosa de gran interés para la salud pública y laboral, dado que, según la Organización Mundial de la Salud, 10 millones de personas en el mundo la contrajeron en el año 2017 y durante este mismo periodo 65 países notificaron 9.299 casos de tuberculosis en trabajadores de la salud, lo cual indica que la tasa de incidencia por tuberculosis en el personal del sector salud fue más del doble que en la población general adulta (1). En Colombia, durante el año 2016, se notificaron 14.480 casos de tuberculosis; 1.135 (7,8\%) de ellos en la ciudad de Bogotá y uno de los grupos más afectados fue el de los trabajadores de la salud (2). En Bogotá, durante el 2014 se notificaron 40 casos de tuberculosis en trabajadores sanitarios; en el 2015 fueron 27; en el 2016 fueron 23 y en el 2017 fueron 19 (3).

El personal de salud es un grupo vulnerable ante la tuberculosis por la exposición a factores de riesgo ocupacionales, entre los cuales se destacan la concentración de partículas infecciosas en el lugar del trabajo, el tiempo de exposición (superior a las seis horas diarias), la sobrecarga laboral, el trabajo nocturno y el desconocimiento de los trabajadores de la salud frente a su exposición ocupacional y las medidas de control de la infección por tuberculosis en las instituciones de salud $(4,5)$.

Sumado a lo anterior, las condiciones de trabajo en el sector de salud evidencian laboral como consecuencia de los pro-

cesos de globalización y neoliberalismo (6), expresadas en multiempleo, contratos inestables y temporales, predominio de contratación a través de cooperativas de trabajo asociado y ausencia de prestaciones sociales. Todo lo anterior contribuye al desgaste del trabajador, al aumento de la susceptibilidad a infección por contaminantes biológicos, como el Mycobacterium tuberculosis y a la disminución de la calidad de la atención en salud prestada (7).

En este contexto, la Organización Panamericana de la Salud y el Centro de Control de Enfermedades de Atlanta (CDC, por sus siglas en inglés) plantearon la necesidad de adoptar directrices relacionadas con las medidas de control administrativas, ambientales y de protección respiratoria de la infección por tuberculosis en las instituciones de salud. Las medidas incluyen un plan institucional de tuberculosis, intervenciones educativas a los trabajadores de la salud sobre la temática e implementar sistemas de vigilancia ocupacional sobre la exposición a Mycobacterium tuberculosis. Las medidas ambientales se componen de la ventilación natural y mecánica y de sistemas complementarios, como el uso de filtros de partícula de alta eficiencia (HEPA, por sus siglas en inglés) y luz ultravioleta germicida (LUV). Las medidas de control de protección respiratoria contemplan el uso adecuado de la mascarilla de alta eficiencia N95 y pruebas cualitativas y cuantitativas de ajuste de este elemento de protección personal $(8,9)$.

La intervención educativa se caracteriza por tener un agente educador (profesionales de la salud) y un propósito 
(fortalecer los conocimientos y prácticas sobre las medidas de control de tuberculosis), se desarrolla en orden para lograr una meta y los acontecimientos (módulos educativos) se vinculan intencionalmente (10). Por otra parte, la educación para adultos busca transformar prácticas en todo momento y lugar, de manera que requiere mantener procesos educativos constantes, favorecer la participación activa de los trabajadores y suministrar reconocimientos por las competencias educativas adquiridas, con el fin de que los trabajadores utilicen los conocimientos adquiridos mediante diversos procesos educativos (11).

Por lo tanto, el presente estudio tuvo como objetivo describir los conocimientos y las prácticas sobre las medidas de control de infección por tuberculosis en trabajadores de la salud antes y después de una intervención educativa.

\section{Materiales y métodos}

Estudio de tipo evaluativo con modelo antes y después (12). Se diseñó un formulario de recolección de datos sobre conocimientos y prácticas frente a las medidas de control de la infección por tuberculosis en instituciones de salud a partir de la revisión de literatura científica y el juicio de expertos. Posteriormente, se realizó una prueba piloto con 30 trabajadores de la salud para verificar su comprensibilidad y se desarrollaron ajustes semánticos, buscando mejor comprensibilidad del formulario de recolección de datos (13).

El pre-test del formulario de recolección de datos fue aplicado a los traba- jadores de la salud de la institución de salud, objeto de estudio por profesionales de enfermería, especialistas en salud y seguridad en el trabajo, entre junio y agosto del 2017. Los trabajadores de la salud cumplieron como criterios de inclusión el estar contratados por prestación de servicios o término indefinido, aceptar participar voluntariamente en la investigación por medio de un consentimiento informado y aceptar participar posteriormente en la intervención educativa. Se excluyeron a los estudiantes, dado que no se podía garantizar su continuidad en la investigación. Los investigadores fueron servicio por servicio en la institución buscando que participara la mayor cantidad de trabajadores de la salud.

A partir de los resultados obtenidos en la aplicación del pre-test a los trabajadores de la salud, se diseñó una intervención educativa con dos módulos, los cuales fueron desarrollados entre diciembre del 2017 y enero del 2018. El diseño de la intervención educativa se sustentó en la andragogía, en la cual se incluye el uso de ayudas y técnicas educativas adaptadas al contexto de los adultos y se tienen en cuenta la experiencia, la interacción grupal y la didáctica (14).

Los dos módulos educativos se realizaron servicio por servicio en la institución de salud objeto de estudio, con un promedio de cuatro trabajadores y duraban entre 30 y 40 minutos. En el módulo 1 se incluyeron contenidos temáticos sobre la etiología de la tuberculosis, signos y síntomas, diagnóstico y tratamiento de esta enfermedad, estrategia de higiene de la tos, definición de 
sintomático respiratorio, la importancia de la captación temprana y el concepto de paciente infeccioso de tuberculosis. Se diseñó y utilizó material educativo audiovisual e impreso, como una presentación animada, fichas sobre la tuberculosis, infografía de higiene de la tos y una estrategia lúdica para retroalimentar la información. Se implementaron técnicas educativas expositivas y de análisis, las cuales se sustentaron en evidencia científica (15).

En el módulo educativo 2 se incluyeron contenidos temáticos sobre las medidas administrativas, ambientales y de protección personal de la infección por tuberculosis. Se elaboró material impreso y visual como folletos sobre las medidas de control de tuberculosis, infografías sobre ventilación natural cruzada y uso de la mascarilla N95 y un calendario sobre medidas de control de tuberculosis. Cabe mencionar que las ayudas educativas utilizadas en los dos módulos (trece fichas, tres folletos, tres infografías y un calendario) fueron realizadas por un diseñador gráfico, con la orientación del equipo investigador y soportadas en evidencia científica. Las ayudas educativas fueron revisadas $y$ validadas por expertos en la temática de estudio.

Al finalizar las sesiones educativas se aplicó un pos-test con las mismas preguntas del formulario de recolección de datos utilizado en el pre-test a los trabajadores que habían participado en esa etapa y en la intervención educativa. Es importante mencionar que el tiempo

86 entre el cual el trabajador respondía el pre-test y el pos-test fue de seis meses.
Se destaca que no existe un tiempo estipulado, en tanto que este depende de la variable que se pretenda medir, en este caso se estableció un intervalo de seis meses entre la aplicación del pre-test y el pos-test (12).

Los datos fueron analizados en SPSS versión 25, se utilizaron estadísticos descriptivos y la prueba de Kolmogorov-Smirnov y teniendo en cuenta que los resultados de esta prueba mostraron una distribución anormal, se realizó la prueba no paramétrica de McNemar para muestras relacionadas o dependientes. El nivel de significancia de alfa fue 0,05 (16).

De la misma manera se obtuvo el aval del comité de ética de la Facultad de Enfermería de la Universidad Nacional de Colombia; se aplicó el consentimiento informado en el pre-test y en el pos-test, y se cumplieron los principios éticos de respeto, confidencialidad y dignidad promulgados en la Declaración de Helsinki (17); de acuerdo con el Ministerio de Salud y Protección Social de la República de Colombia, la investigación fue clasificada en la categoría sin riesgo, dado que no se modificaron variables biológicas, fisiológicas, psicológicas o sociales de los participantes (18).

\section{Resultados}

En el pre-test participaron 442 trabajadores de la institución de salud objeto de estudio, de los cuales 216 participaron en los dos módulos de la intervención educativa. De los 216 trabajadores, $40(18,5 \%)$ fueron hombres y 176 
$(81,5 \%)$ mujeres, $112(51,9 \%)$ tenían de posgrado. En la tabla 1 se describen un nivel educativo de técnico/tecnólogo, algunas variables ocupacionales de los $79(36,6 \%)$ universitario y $25(11,6 \%)$ participantes.

Tabla 1. Descripción de variables ocupacionales de los trabajadores que participaron en el estudio (Bogotá, 2017)

\begin{tabular}{|c|c|c|}
\hline Variable & $\mathbf{N}$ & $\%$ \\
\hline \multicolumn{3}{|l|}{ Ocupación } \\
\hline Médico & 24 & 11,1 \\
\hline Enfermero & 46 & 21,3 \\
\hline Bacteriólogo & 8 & 3,7 \\
\hline Auxiliar de enfermería & 76 & 35,2 \\
\hline Auxiliar de laboratorio & 10 & 4,6 \\
\hline Médico especialista & 19 & 8,8 \\
\hline Administrativo & 6 & 2,8 \\
\hline Terapeuta respiratorio & 5 & 2,3 \\
\hline Camillero & 22 & 10,2 \\
\hline \multicolumn{3}{|c|}{ Tiempo que ha ejercido su ocupación } \\
\hline Menor a 2 años & 33 & 15,3 \\
\hline 2-5 años & 70 & 32,4 \\
\hline 5-10 años & 75 & 34,7 \\
\hline Mayor a 10 años & 38 & 17,6 \\
\hline \multicolumn{3}{|l|}{ Servicio o área de trabajo } \\
\hline Urgencias & 57 & 26,4 \\
\hline Hospitalización & 88 & 40,7 \\
\hline Laboratorio & 13 & 6 \\
\hline Unidad de cuidado intensivo & 38 & 17,6 \\
\hline Consulta externa & 20 & 9,3 \\
\hline
\end{tabular}

Fuente: elaboración propia. 
Para el análisis de los datos se utilizó la prueba no paramétrica de McNemar para muestras relacionadas o dependientes (pre-test y pos-test), encontrando valores de $\mathrm{P}$ menores a 0,05 (ver tabla 2 ), lo que sugiere mejores conocimientos y prácticas frente a las medidas de control administrativas, ambientales y de protección personal de la infección por tuberculosis, debido a la educación realizada en los trabajadores. En la misma tabla se presenta la distribución de frecuencias y porcentajes de cada ítem del formulario de recolección de datos en el pre-test y en el pos-test, así como los resultados del valor de $\mathrm{P}$ de la prueba no paramétrica de McNemar para muestras relacionadas o dependientes.

Tabla 2. Distribución de frecuencias y porcentajes relacionados con los conocimientos y las prácticas sobre las medidas administrativas, ambientales y de protección personal de la infección por tuberculosis de los trabajadores que participaron (Bogotá, 2017)

\begin{tabular}{|c|c|c|c|c|c|}
\hline & \multicolumn{2}{|c|}{ Pretest $\mathrm{N}=216$} & \multicolumn{2}{|c|}{ Postest $\mathrm{N}=216$} & \\
\hline $\begin{array}{l}\text { Contenido } \\
\text { temático }\end{array}$ & $\begin{array}{c}\mathrm{N}(\%) \\
\text { conocimientos } \\
\text { y prácticas } \\
\text { correctos }\end{array}$ & $\begin{array}{c}\mathrm{N}(\%) \\
\text { conocimientos } \\
\text { y prácticas } \\
\text { incorrectos }\end{array}$ & $\begin{array}{c}\mathrm{N}(\%) \\
\text { conocimientos } \\
\text { y prácticas } \\
\text { correctos }\end{array}$ & $\begin{array}{c}\mathrm{N}(\%) \\
\text { conocimientos } \\
\text { y prácticas } \\
\text { incorrectos }\end{array}$ & $\mathbf{P}$ \\
\hline
\end{tabular}

Medidas administrativas del control de la infección por tuberculosis

\begin{tabular}{|c|c|c|c|c|c|}
\hline $\begin{array}{l}\text { 1. Prueba } \\
\text { cutánea de } \\
\text { tuberculina }\end{array}$ & $162(75 \%)$ & $54(25 \%)$ & $212(98,1 \%)$ & $4(1,9 \%)$ & 0 \\
\hline $\begin{array}{l}\text { 2. Sintomático } \\
\text { respiratorio }\end{array}$ & $181(83,8 \%)$ & 35 (16,2\%) & $215(99,5 \%)$ & $1(0,5 \%)$ & 0 \\
\hline $\begin{array}{l}\text { 3. Signos y } \\
\text { síntomas más } \\
\text { frecuentes de } \\
\text { la tuberculosis } \\
\text { pulmonar } \\
\text { activa }\end{array}$ & $208(96,3 \%)$ & $8(3,7 \%)$ & $215(99,5 \%)$ & $1(0,5 \%)$ & 0,039 \\
\hline $\begin{array}{l}\text { 4. Aislamiento } \\
\text { a pacientes } \\
\text { infecciosos de } \\
\text { tuberculosis }\end{array}$ & $150(69,4 \%)$ & $66(30,6 \%)$ & $209(96,8 \%)$ & $7(3,2 \%)$ & 0 \\
\hline $\begin{array}{l}\text { 5. Paciente } \\
\text { infeccioso de } \\
\text { tuberculosis }\end{array}$ & $179(82,9 \%)$ & $37(17,1 \%)$ & $198(91,7 \%)$ & $18(8,3 \%)$ & 0,012 \\
\hline $\begin{array}{l}\text { 6. Captación de } \\
\text { sintomáticos } \\
\text { respiratorios } \\
\text { institucional }\end{array}$ & $38(17,6 \%)$ & $178(82,4 \%)$ & $146(67,6 \%)$ & $70(32,4 \%)$ & 0 \\
\hline $\begin{array}{l}\text { 7. Ubicación de } \\
\text { sintomáticos } \\
\text { respiratorios }\end{array}$ & $115(53,2 \%)$ & $101(46,8 \%)$ & $183(84,7 \%)$ & $33(15,3 \%)$ & 0 \\
\hline
\end{tabular}


Continuación Tabla 2. Distribución de frecuencias y porcentajes relacionados con los conocimientos y las prácticas sobre las medidas administrativas, ambientales y de protección personal de la infección por tuberculosis de los trabajadores que participaron (Bogotá, 2017)

\begin{tabular}{c|c|c|c|c|c}
\hline & \multicolumn{2}{|c|}{ Pretest $\mathrm{N}=216$} & \multicolumn{2}{c|}{ Postest $\mathrm{N}=216$} & \\
\hline \multirow{2}{*}{$\begin{array}{c}\text { Contenido } \\
\text { temático }\end{array}$} & $\begin{array}{c}\mathrm{N}(\%) \\
\text { conocimientos } \\
\text { y prácticas } \\
\text { correctos }\end{array}$ & $\begin{array}{c}\mathrm{N}(\%) \\
\text { conocimientos } \\
\text { y prácticas } \\
\text { incorrectos }\end{array}$ & $\begin{array}{c}\mathrm{N}(\%) \\
\text { conocimientos } \\
\text { y prácticas } \\
\text { correctos }\end{array}$ & $\begin{array}{c}\mathrm{N}(\%) \\
\text { conocimientos } \\
\text { y prácticas } \\
\text { incorrectos }\end{array}$ & $\mathrm{P}$ \\
\hline
\end{tabular}

Medidas ambientales del control de la infección por tuberculosis

\begin{tabular}{|c|c|c|c|c|}
\hline $\begin{array}{l}\text { 8. Ventilación } \\
\text { natural cruzada }\end{array}$ & $131(60,6 \%)$ & 85 (39,4\%) & $210(97,2 \%)$ & $6(2,8 \%)$ \\
\hline $\begin{array}{l}\text { 9.Implementación } \\
\text { de la ventilación } \\
\text { cruzada natural }\end{array}$ & $92(42,6 \%)$ & $124(57,4 \%)$ & $155(71,8 \%)$ & $61(28,2 \%)$ \\
\hline $\begin{array}{l}\text { 10. Ventilación } \\
\text { mecánica }\end{array}$ & $144(66,7 \%)$ & 72 (33,3\%) & 207 (95,8\%) & $9(4,2 \%)$ \\
\hline $\begin{array}{l}\text { 11. Filtros de } \\
\text { partícula de } \\
\text { alta eficiencia } \\
\text { (HEPA) }\end{array}$ & $40(18,5 \%)$ & $176(81,5 \%)$ & $201(93,1 \%)$ & $15(6,9 \%)$ \\
\hline $\begin{array}{l}\text { 12. Luz } \\
\text { ultravioleta } \\
\text { germicida }\end{array}$ & $78(36,1 \%)$ & $138(63,9 \%)$ & $204(94,4 \%)$ & $12(5,6 \%)$ \\
\hline $\begin{array}{l}\text { 13. Precauciones } \\
\text { con la luz } \\
\text { ultravioleta } \\
\text { germicida }\end{array}$ & $70(32,4 \%)$ & $146(67,6 \%)$ & 207 (95,8\%) & $9(4,2 \%)$ \\
\hline
\end{tabular}

\section{Medidas de protección personal del control de la infección por tuberculosis}

\begin{tabular}{|c|c|c|c|c|c|}
\hline $\begin{array}{l}\text { 14. Mascarilla } \\
\text { de alta } \\
\text { eficiencia N95 }\end{array}$ & $157(72,7 \%)$ & $59(27,3 \%)$ & $213(98,6 \%)$ & $3(1,4 \%)$ & 0 \\
\hline $\begin{array}{l}\text { 15. Pruebas } \\
\text { cualitativas de } \\
\text { ajuste de la } \\
\text { mascarilla de } \\
\text { alta eficiencia } \\
\text { N95 }\end{array}$ & 30 (13,9\%) & $186(86,1 \%)$ & $200(92,6 \%)$ & $16(7,4 \%)$ & 0 \\
\hline $\begin{array}{l}\text { 16.Implementación } \\
\text { de los pasos } \\
\text { adecuados } \\
\text { para el uso de } \\
\text { la mascarilla de } \\
\text { alta eficiencia } \\
\text { N95 }\end{array}$ & $112(51,9 \%)$ & $104(48,1 \%)$ & $206(95,4 \%)$ & $10(4,6 \%)$ & U \\
\hline
\end{tabular}

Fuente: elaboración propia. 
Es importante mencionar que algunos trabajadores de la salud describieron en el espacio de observaciones de los formularios de recolección de datos del pre-test y del pos-test los siguientes aspectos: la institución no realiza la prueba de tuberculina para detectar tuberculosis latente en el marco de un sistema de vigilancia ocupacional; los trabajadores refirieron que no tenían la posibilidad de ubicar a los pacientes sintomáticos respiratorios en un lugar diferente al de otros pacientes e implementar la ventilación natural cruzada, debido a la infraestructura de la institución de salud; también se mencionó que la institución no dispone de forma continua de la mascarilla de alta eficiencia N95 y que nunca se realizaban pruebas cualitativas de la mascarilla N95.

\section{Discusión}

Se identificó que aumentaron los conocimientos de los trabajadores de la salud en relación a: concepto de sintomático respiratorio $(\mathrm{P}<0,05)$, reconocer los signos y los síntomas de la tuberculosis pulmonar $(\mathrm{P}=0,039)$ y el tipo de aislamiento a pacientes con tuberculosis infecciosa $(\mathrm{P}<0,05)$. Al respecto, un estudio desarrollado en Malawi (África) señala que es indispensable que los trabajadores de la salud reconozcan los signos y los síntomas de la tuberculosis y el tipo de aislamiento, pues así detectan rápidamente los sintomáticos respiratorios y logran aislarlos, lo cual contribuye al control de la tuberculosis en las instituciones de salud (19).

90 Se encontró que con la intervención educativa aumentaron los conocimien- tos sobre captación de sintomáticos respiratorios $(\mathrm{P}<0,05)$ y la ubicación de estos en un lugar diferente al de otros pacientes para evitar el contagio $(\mathrm{P}<$ $0,05)$. Se destaca que las limitaciones de la infraestructura en las instituciones de salud se convierten en una barrera en la implementación de las medidas de control, dado que las fallas en el diseño de la infraestructura de las instituciones no permiten realizar triage o una ubicación de los sintomáticos respiratorios en otras áreas, lo cual es un aspecto que limita el corte de la cadena de transmisión de la enfermedad en los establecimientos sanitarios (20).

Así mismo, los conocimientos sobre qué es la prueba de tuberculina aumentaron en los trabajadores de la salud ( $\mathrm{P}$ $<0,05)$; no obstante, la institución no realiza esta prueba para determinar la infección tuberculosa latente. La prueba de tuberculina es crucial para el control de la tuberculosis en los trabajadores de la salud, en tanto que permite detectar $y$ monitorear la incidencia y la prevalencia de tuberculosis latente en los trabajadores $(21,22)$.

En relación con los conocimientos sobre medidas de tipo ambiental de la infección por tuberculosis, estos aumentaron significativamente, igual a lo referido en relación a las prácticas sobre la ventilación natural cruzada $(\mathrm{P}<0,05)$; aun así, en algunos servicios no es posible implementar esta medida por la falta de puertas y ventanas. $\mathrm{Al}$ respecto, algunos estudios han señalado que la ventilación natural es una medida costo-efectiva para el control de la tuberculosis en instituciones de salud con recursos limita- 
dos, ya que optimiza el diseño de las edificaciones; sin embargo, en ocasiones las instituciones de salud no cuentan con las mínimas condiciones para la adopción de estas medidas. La ventilación natural se facilita en instituciones de salud ubicadas en climas cálidos, pero en las ubicadas en climas fríos la LUV puede producir mayores beneficios $(23,24)$.

Es importante mencionar que el primer paso para utilizar la ventilación natural es evaluar el flujo direccional del aire, el cual debe ir de las zonas limpias a las zonas menos limpias. Sumado a que, la ventilación natural depende de las condiciones climáticas exteriores y que las dos fuerzas que generan el flujo de aire son el viento y la temperatura, de manera que la ventilación natural se encuentra sujeta a variaciones estocásticas y, por tanto, se requiere evaluar la posibilidad de su implementación en las instituciones de salud (25).

En cuanto a las medidas de protección personal de la infección por tuberculosis, se encontró un aumento en relación a las respuestas sobre el conocimiento de mascarilla de alta eficiencia N95 (P $0<0,05)$ y sobre la implementación de los pasos recomendados por el CDC de Atlanta para el uso de este elemento de protección personal $(\mathrm{P}<0,05)$.

$\mathrm{Al}$ respecto, un estudio ejecutado en $\mathrm{Su}-$ ráfrica evidenció que los trabajadores que fueron capacitados sobre el uso de la mascarilla de alta eficiencia N95 tenían entre 36 y 40 veces más probabilidades de usar este elemento de protección personal para prevenir el contagio por tuberculosis (26); los trabajadores de la salud que usan adecuadamente la mascarilla de alta eficiencia N95 presentan bajas tasas de infección y colonización por agentes biológicos (27), a pesar de las molestias y la incomodidad que puede generar, entre las cuales se destaca la presión sobre la nariz, la dificultad para respirar, la cefalea y el aumento de la temperatura facial (28).

La prueba de ajuste es un mecanismo de evaluación de la capacidad de hermeticidad entre los bordes de la mascarilla N95 y la superficie facial del trabajador; las pruebas cualitativas se basan en la percepción de olores por el trabajador mientras se realizan algunos movimientos corporales y las pruebas cuantitativas evalúan la concentración de partículas dentro y fuera de la mascarilla N95 al medir el valor de factor de ajuste $(29,30)$.

En el presente estudio, la intervención educativa contribuyó en el aumento de los conocimientos en relación a las pruebas cualitativas de la mascarilla de alta eficiencia N95 $(\mathrm{P}<0,05)$. Una investigación desarrollada en Nigeria menciona que las instituciones de salud no realizan las pruebas cuantitativas o cualitativas de la mascarilla de alta eficiencia N95, mientras que los trabajadores de la salud refieren que este elemento de protección personal no se usa rutinariamente en la atención de pacientes con sospecha o diagnóstico de tuberculosis (31).

Como limitaciones en el presente estudio se destaca que los estudios evaluativos con modelo antes y después, sin grupo de comparación, puede que no eliminen el efecto del contexto. Por lo tanto, a futuro se recomienda el desarro- 
llo de otros estudios de mayor alcance con grupos de comparación que contemplen métodos experimentales y cuasi experimentales, con el fin de mitigar el efecto del contexto (12).

Igualmente, otra limitación que presentó esta investigación hace referencia a la alta rotación del personal relacionada con las formas de contratación laboral (la mayoría por contrato civil por prestación de servicio) que se dan en el sector salud, lo que puede incidir en que el número de trabajadores que participaron en el pretest fue superior comparado a los que participaron en el postest.

En Colombia, los estudios realizados sobre los conocimientos y las prácticas de los trabajadores de la salud frente a las medidas de control de la infección por tuberculosis son escasos. No obstante, un estudio identificó la importancia de realizar intervenciones educativas con los trabajadores de la salud sobre qué es un sintomático respiratorio, el número de muestras de esputo, los signos y los síntomas de la tuberculosis pulmonar activa y el diagnóstico de esta enfermedad (32). También otro estudio bibliométrico destaca la importancia de abordar la tuberculosis de origen ocupacional en Colombia, los factores de riesgo ocupacional asociados al contagio, la implementación de la ventilación natural y mecánica y el uso de mascarillas N95 (33).

Un estudio de revisión realizado en Colombia identificó tres tipos de medidas de control de la infección por tuberculosis: las administrativas, relacionadas

92 con la detección oportuna de sintomáticos respiratorios, casos de tuberculosis e inicio oportuno del tratamiento para minimizar los riesgos de transmisión e infección; las ambientales, destinadas a dar buen uso a la ventilación natural y en algunos casos utilizar LUV y filtros de alta eficiencia, y las de protección respiratoria, sobre el uso de la mascarilla de alta eficiencia N-95 (34).

Así mismo, en Colombia se realizó un estudio para evaluar la implementación de medidas de control de tipo administrativo, ambiental y de protección respiratoria de la infección por tuberculosis en una institución de salud de Bogotá. Se encontró frente a las medidas administrativas que la institución de salud no tenía definido un plan institucional ni un comité responsable del control de la infección de tuberculosis; sobre las medidas de control ambientales se evidenció que la institución solo contaba con sistemas de ventilación natural, excepto en los servicios de reanimación y laboratorio clínico, los cuales tenían sistemas de extracción y recirculación de aire y presentaban dificultades por déficit de mantenimiento; en las medidas de protección respiratoria se identificó que la institución suministraba permanentemente a los trabajadores de la salud mascarillas quirúrgicas; no obstante, la dotación de la mascarilla de alta eficiencia N-95 estaba sujeta a procesos de solicitud y autorización de insumos, puesto que el suministro de este elemento de protección personal se produce después del diagnóstico y no desde el momento de ingreso del paciente infeccioso de tuberculosis (20). También en otra investigación realizada en este mismo país con el objetivo de describir la implementación de las medidas de control de la infección por 
tuberculosis en 51 instituciones de salud, se identificó que solamente $35(68,6 \%)$ instituciones de salud contaban con plan escrito para el control de tuberculosis, tan solo $36(70,5 \%)$ realizan capacitaciones a los trabajadores de la salud sobre tuberculosis, solo $8(16,3 \%)$ tenían un monitoreo diario del aire natural o mecánico y $43(84,3 \%)$ tenían mascarillas de alta eficiencia N95 o FFP2 disponibles para los trabajadores de la salud, no obstante solamente $35(68,6 \%)$ habían entrenado a sus trabajadores de la salud en el uso y en la apropiada colocación de las mascarillas de alta eficiencia N95 (24).

Se destaca que todos estos estudios han sido desarrollados por el Grupo de Investigación de Salud y Cuidado de los Colectivos de la Facultad de Enfermería de la Universidad Nacional de Colombia que, a su vez y dado el avance de las investigaciones realizadas, identificó la necesidad de realizar un estudio de mayor alcance e impacto sobre los conocimientos y las prácticas de los trabajadores de la salud sobre las medidas de control de la infección por tuberculosis, en tanto que los estudios previos realizados en Colombia eran de alcance descriptivo. Aunque se reconoce el avance, también es claro que es necesario seguir afinando aspectos metodológicos y psicométricos.

A modo de conclusión, la intervención educativa mejoró los conocimientos y las prácticas de los trabajadores de la salud frente a las medidas de control administrativas, ambientales y de protección personal de la infección por tuberculosis. Así, intervenciones educativas con ayudas y técnicas pedagógicas pueden contribuir en la protección de los trabajadores de la salud en relación a su exposición ocupacional al Mycobacterium tuberculosis.

Por otra parte, se requiere el desarrollo de políticas públicas que contribuyan al monitoreo de la implementación de las medidas de control de infección de tuberculosis en las instituciones de salud. Así mismo, las instituciones deben asegurar procesos que incluyan la identificación, la notificación, el monitoreo, la interpretación y el análisis de casos de tuberculosis en trabajadores de la salud para la toma de decisiones, así como la implementación de directrices para la adopción de las medidas de control de infección por tuberculosis con asignación de recursos que garanticen la sostenibilidad de las mismas.

\section{Agradecimientos}

A los trabajadores de la salud de la institución de salud participante, por su apoyo y compromiso en el desarrollo del proyecto de investigación.

\section{Financiación}

El proyecto de investigación "Intervención Educativa sobre las Medidas de Control por Tuberculosis en Trabajadores de la Salud de una Institución de Salud de Bogotá D.C. 2017-2018”, con código Hermes 37270, el cual fue financiado por la Universidad Nacional de Colombia.

\section{Conflicto de intereses}

Los autores declaramos que no existió conflicto de intereses. 


\section{Referencias bibliográficas}

1. World Health Organization. Global Tuberculosis Report 2018 [Internet]. Geneva: World Health Organization; 2018 [citado 2018 sept. 24]. Disponible en: http:// apps.who.int/iris/bitstream/handle/10665/ 274453/9789241565646-eng.pdf?ua=1

2. Instituto Nacional de Salud. Informe Tuberculosis Colombia 2017 [Internet]. Colombia. 2017 [citado 2018 sept. 24]. Disponible en: https://www.ins.gov.co/ buscador-eventos/Informesdeevento/Tuberculosis\%202017.pdf

3. Secretaría Distrital de Salud de Bogotá D.C. Programa de Control de Tuberculosis, 2017. Datos preliminares. Bogotá: Secretaría Distrital de Salud de Bogotá; 2017.

4. Zhou F, Zhang L, Gao L, Hao Y, Zhao $X$, Liu J, et al. Latent tuberculosis infection and occupational protection among health care workers in two types of public hospitals in China. PLoS One. 2014;9:1-8. Doi: 10.1371/journal.pone.0104673

5. Nienhaus A, Schablon A, Preisser AM, Ringshausen FC, Diel R. Tuberculosis in healthcare workers -a narrative review from a German perspective-. J Occup Med Toxicol. 2014;9:1-12. Doi: 10.1186/1745-6673-9-9

6. Feo O. Reflexiones sobre la globalización y su impacto sobre la salud de los trabajadores y el ambiente. Ciênc. saúde coletiva. 2003;8(4):887-96. Doi: 10.1590/ S1413-81232003000400011

7. Organización Panamericana de la Salud, Ministerio de Salud de Brasil y Universidad Federal de Minas Gerais. Condiciones de Salud y Trabajo en el Sector Salud [Internet]. 2008 [citado 2018 mzo. 12]. Disponible en: http://www.observatoriorh.org/sites/default/files/webfiles/fulltext/cst_sector_salud.pdf

8. Organización Panamericana de la Salud. Lineamientos para la implementación en las Américas [Internet]. 2014 [citado

2018 febr. 9]. Disponible en: http://www. paho.org/hq/index.php?option=com docman\&task=doc_view\&gid $=25787 \&$ Itemi$d=270$ \&lang $=$ en

9. Centers for Disease Control and Prevention (CDC). Tuberculosis elimination infection control in health-care settings [Internet]. 2012 [citado 2018 febr. 15]. Disponible en: http://www.cdc.gov/tuberculosis/publications/factsheets/prevention/ ichcs.pdf

10. Touriñan JM. Intervención educativa, intervención pedagógica y educación: La mirada pedagógica. Revista portuguesa de pedagogía [Internet]. 2011 [citado 2018 mzo. 17];1(1):283-307. Disponible en: https://www.liberquare.com/blog/content/ intervencioneducativa.pdf

11. Martínez J. ¿Qué es la educación de adultos?: Responde la Unesco [Internet]. San Sebastián: Editorial Centro Unesco; 2006 [citado 2018 febr. 15]. Disponible en: http://unesdoc.unesco.org/images/0014/001494/149413s.pdf

12. Vega R, Torres T, Cerna R. Revisión documental acerca de la investigación evaluativa [Internet]. Contribuciones a las Ciencias Sociales. 2013 [citado 2018 mzo. 10]. Disponible en: http:// www.eumed.net/rev/cccss/23/investigacion-evaluativa-politicas-publicas-mexico.html

13. Sánchez R, Echeverry J. Validación de escalas de medición en salud. Rev Salud Pública [Internet]. 2004 [citado 2018 mzo. 15];6:302-18. Disponible en: https://www.scielosp.org/article/rsap/2004. v6n3/302-318/

14. Sanz F, Lancho J, Guil R, Gómez V, Luna R. Fundamentos de la educación de personas adultas. España: Instituto de Tecnologías Educativas, Ministerio de Educación; 2010 [citado 2018 febr. 20]. Disponible en: http://www.ite.educacion.es/formacion/materiales/125/cd/unidad_1/introduccion.htm 
15. Riquelme-Pérez M. Metodología de educación para la salud. Rev Pediatr Aten Primaria [Internet]. 2012 [citado 2018 febr. 24];14(22):77-82. Doi: 10.4321/ S1139-76322012000200011

16. Berlanga V, Rubio MJ. Clasificación de pruebas no paramétricas. Cómo aplicarlas en SPSS. Revista de Investigación e Innovación Educativa [Internet]. 2012 [citado 2018 febr. 24];5(2):101-13. Doi: 10.1344/reire2012.5.2528

17. Asociación Médica Mundial. Declaración de Helsinki de la AMM - Principios éticos para las investigaciones médicas en seres humanos [Internet]. 2015 [citado 2018 mzo. 20]. Disponible en: https:// www.wma.net/es/policies-post/declaracion-de-helsinki-de-la-amm-principios-eticos-para-las-investigaciones-medicas-en-seres-humanos/

18. Ministerio de Salud y Protección Social de la República de Colombia. Resolución 8430 de 1993, "Por la cual se establecen las normas científicas, técnicas y administrativas para la investigación en salud" [Internet]. Colombia; 1993 [citado 2018 mzo. 20]. Disponible en: https:// www.minsalud.gov.co/sites/rid/Lists/BibliotecaDigital/RIDE/DE/DIJ/RESOLUCION-8430-DE-1993.PDF

19. Flick RJ, Munthali A, Simon K, Hosseinipour M, Kim MH, Mlauzi L, et al. Assessing infection control practices to protect health care workers and patients in Malawi from nosocomial transmission of Mycobacterium tuberculosis. PLoS One. 2017;12(12):e0189140. Doi: 10.1371/ journal.pone.0189140

20. Muñoz Al, Castro Y. Medidas de control de tuberculosis en una institución de salud de Bogotá D.C. Rev Fac Nac Salud Pública [Internet]. 2016 [citado 2018 febr. 27];34(1):38-47. Doi: 10.17533/udea. rfnsp.v34n1a05

21. Basavaraj A, Chandanwale A, Patil A, Kadam D, Joshi S, Gupte N, et al. Tuberculosis risk among medical trainees, Pune, India. Emerg Infect Dis [Internet].
2016 [citado 2018 febr. 22];22(3):541-3. Doi: 10.3201/eid2203.151673

22. Vidella JS, Catalan T, Callau C, Lejeune M. Infección tuberculosa latente en trabajadores sanitaros de un hospital general básico en Cataluña. Arch Prev Riesgos Labor [Internet]. 2014 [citado 2018 febr. 28];17(2):97-101. Disponible en: https://dialnet.unirioja.es/servlet/ articulo?codigo $=4712305$

23. Nathavitharana RR, Bond $P$, Dramowski A, Kotze K, Lederer P, Oxley I, et al. Agents of change: The role of healthcare workers in the prevention of nosocomial and occupational tuberculosis. Presse Med [Internet]. 2017 [citado 2018 febr. 26];46(2):e53-e62. Doi: 10.1016/j. Ipm.2017.01.014

24. Muñoz A, Saavedra C, Cruz O. Control de la infección por tuberculosis en instituciones de salud. Rev Investigaciones Andinas [Internet]. 2017 [citado 2018 mzo. 17];33(18):1683-96. Disponible en: http:// revia.areandina.edu.co/ojs/index.php/IA/ article/view/653/587

25. Organización Panamericana de la Salud. Ventilación natural para el control de las infecciones en entornos de atención de la salud [Internet]. Washington; 2009 [citado 2018 mzo. 9]. Disponible en: http://www1.paho.org/hq/ dmdocuments/2011/ventilacion_natual_ spa_25mar11.pdf

26. Malotle MM, Spiegel JM, Yassi A, Ngubeni D, O'Hara LM, Adu PA, et al. Occupational tuberculosis in South Africa: are health care workers adequately protected? Public Health Action [Internet]. 2017 [citado 2018 febr. 25];7(4):258-67. Doi: 10.5588/pha.17.0070

27. Maclntyre CR, Wang Q, Rahman B, Seale H, Ridda I, Gao Z, et al. Efficacy of face masks and respirators in preventing upper respiratory tract bacterial colonization and co-infection in hospital healthcare workers. Prev Med [Internet]. 2014 [citado 2018 febr. 28];62:1-7. Doi: 10.1016/j. ypmed.2014.01.015 
28. Baig AS, Knapp C, Eagan AE, Radonovich LJ. Health care workers' views about respirator use and features that should be included in the next generation of respirators. Am J Infect Control [Internet]. 2010 [citado 2018 febr. 27];38(1):1825. Doi: 10.1016/j.ajic.2009.09.005

29. Or P, Chung J, Wong T. A novel approach to fit testing the N95 respirator in real time in a clinical setting. International Journal of Nursing Practice [Internet]. 2016 [citado 2018 febr. 28];22(1):22-30. Doi: $10.1111 /$ ijn.12354

30. Ciotti C, Pellissier G, Rabaud C, Lucet JC, Abiteboul D, Bouvet E. Effectiveness of respirator masks for healthcare workers, in France. Med Mal Infect. 2012; 42(6): 264-269. DOI: https://doi.org/10.1016/j. medmal.2012.05.001

31. Dokubo EK, Odume B, Lipke V, Muianga $\mathrm{C}$, Onu E, Olutola A, et al. Building and strengthening infection control strategies to prevent tuberculosis - Nigeria, 2015. The Morbidity and Mortality Weekly Report [Internet]. 2016 [citado 2018 febr.
26];65(10):263-6. Doi: 10.15585/mmwr. mm6510a3

32. Muñoz Al, Puerto AH, Pedraza LM. Intervención educativa en trabajadores de la salud sobre captación de sintomáticos respiratorios de tuberculosis. Rev Cubana Salud Pública [Internet]. 2015 [citado 2019 mzo. 7];41(1):46-56. Disponible en: http:// scielo.sld.cu/scielo.php?script=sci_arttext\&pid=S0864-34662015000100005\&In$\mathrm{g}=\mathrm{es}$.

33. Cruz OA, Muñoz Al. Estudio bibliométrico sobre tuberculosis en trabajadores de la salud. Med Segur Trab [Internet]. 2012 [citado 2019 mzo. 6];58(229):30320. Disponible en: http://scielo.isciii.es/ pdf/mesetra/v58n229/original2.pdf

34. Muñoz Al, Pico CL, Muñoz YL. Control de la tuberculosis en los trabajadores de instituciones hospitalarias. Salud de los trabajadores [Internet]. 2015 [citado 2019 mzo. 7];23(2):13743. Disponible en: http://www.scielo. org.ve/scielo.php?script=sci_arttext\&pi$\mathrm{d}=\mathrm{S} 1315-01382015000200007$ 